\title{
Löffler syndrome caused by extensive cutaneous larva migrans: A case report and review of the literature
}

$M$ te Booij, EMGJ de Jong, HJ Bovenschen

Dermatology Online Journal 16 (10): 2

Department of Dermatology, Radboud University Nijmegen Medical Centre, Nijmegen, The Netherlands. j.bovenschen@derma.umcn.nl

\section{Abstract}

In rare cases, cutaneous larva migrans may be complicated by Löffler syndrome. This syndrome is thought to result from a type I hypersensitivity reaction related to the pulmonary larval migration phase of various parasites. It is characterized by migratory pulmonary eosinophilic infiltrates and peripheral eosinophilia, with malaise, fever, and cough. Our patient was successfully treated with ivermectin, a corticosteroid cream, and inhalation medication in an early phase, which prevented complications. We present the details of this case and review the literature.

\section{Introduction}

Cutaneous larva migrans is a condition caused by the epidermal migration of nematodes. We report a 27-year-old man with a creeping skin eruption after a holiday in Thailand who developed pulmonary symptoms after arriving at home. The diagnosis of cutaneous larva migrans with subsequent Löffler syndrome was made based on the clinical picture, radiographic findings, and blood tests. In only very rare cases, patients develop both cutaneous larva migrans and Löffler syndrome [1]. It is important to diagnose Löffler syndrome at an early stage to prevent unnecessary complications.

\section{Case synopsis}

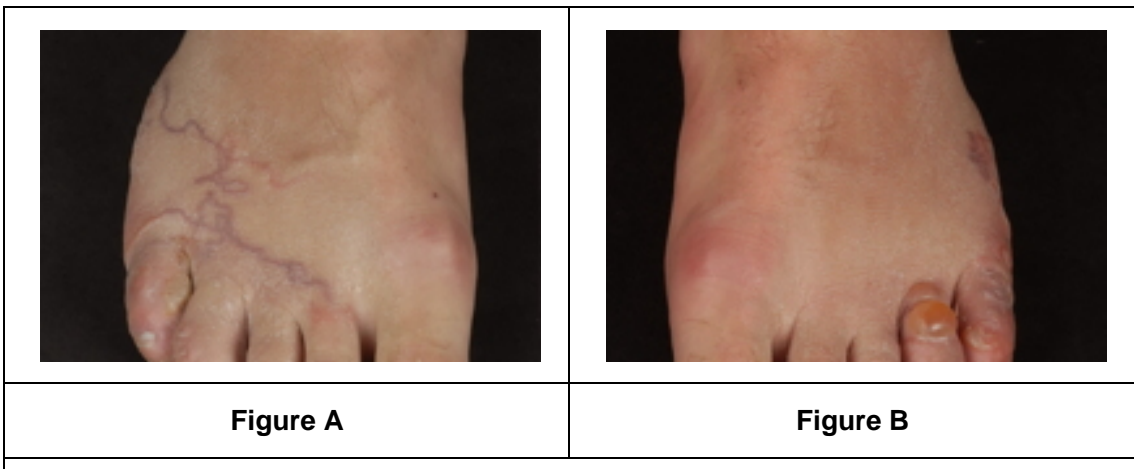

Figure A. Livid erythematous, serpiginous tracks on the right foot, with interdigital tinea pedis.

Figure B. Left foot, with a 'creeping eruption' on the lateral side and with extensive vesicle/bullae formation.

A 27-year-old man, who had just returned from a 2-week beach holiday in Thailand, presented with a 10-day history of an itchy skin eruption on both feet. The symptoms started one week after his arrival in Thailand and were progressive. Clinical examination revealed erythematous/livid serpiginous tracks with erythema, vesicles, and some bullae on the distal part of both feet, together with interdigital maceration. (Figures A, B, and C) The patient had a history of mild asthma (without any medication for 10 years) and recurrent interdigital tinea pedis. 


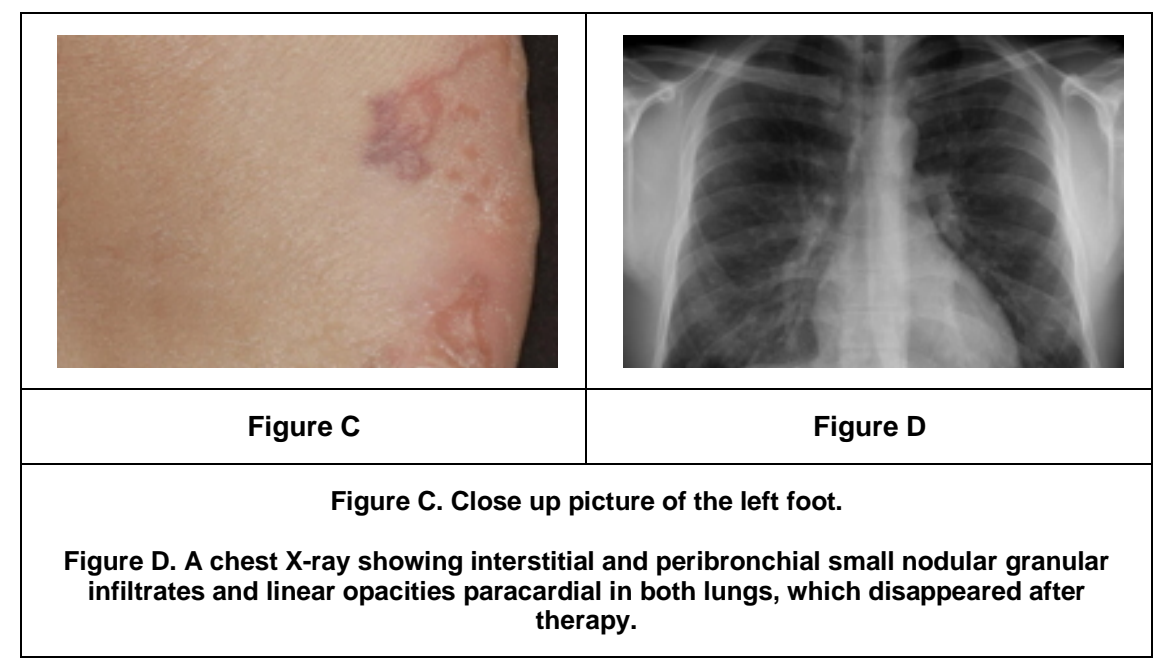

Based on the clinical picture the diagnosis of cutaneous larva migrans with a severe secondary eczematous reaction was made. Because of the severe secondary skin reaction, treatment with clobetasol-17 propionate (Dermovate ${ }^{\circledR}$ ) cream twice daily for 1 week and a single oral dose of $18 \mathrm{mg}(200 \mu \mathrm{g} / \mathrm{kg}$ ) ivermectin (Stromectol $\circledast$ ) was initiated. During one week the skin significantly improved. However, he then presented with an exacerbation of his asthma, which raised a suspicion of an additional pulmonary reaction. Further investigations showed an elevated C-reactive protein (CRP): $35 \mathrm{mg} / \mathrm{l}$, and eosinophilia (differentiation: $21 \%$ $(n=0-6 \%)$, absolute count: $2.70 .109 / /(n=0.03 .109-0.76 .109 / /))$ in peripheral blood. A chest $X$-ray revealed small nodular granular infiltrates and linear paracardial opacities in both lungs, as may be observed in Löffler syndrome (Figure D). Fecal examination was negative for parasites and eggs. The clinical presentation, laboratory results, and radiographic signs led to a diagnosis of Löffler syndrome related to extensive cutaneous larva migrans. Treatment with clobetasol-17 propionate cream, ivermectin, and his inhalation medication (budesonide/formoterol (Symbicort ${ }^{\circledR}$ ) led to complete remission of clinical symptoms and normalization of peripheral blood CRP, eosinophilia, and radiographic abnormalities after 3 months.

\section{Discussion and review of the literature}

Cutaneous larva migrans is a condition caused by the epidermal larval migration of nematodes, most commonly the animal hookworm, Ancylostoma braziliense [2, 3]. This skin infestation typically originates from sub-tropical areas and is endemic in the Caribbean, Central America, South America, Southeast Asia and Africa. Walking barefoot on the beach was a risk factor in 95 percent of all cases. In one study 6.7 percent of travelers visiting a travel-related disease clinic presented with cutaneous larva migrans [2].

Adult hookworms live in the intestines of cats and dogs; the worm eggs are excreted in feces. The eggs survive in wet soil and develop into infective third stage larvae after about one week. After having located a host, larvae search for a suitable penetration site and eventually penetrate the skin [3]. Skin defects such as tinea pedis may facilitate the infiltration, as seen in the present case. However, these predisposing conditions are not obligatory. Several hours after penetration, a red papule or non-specific dermatitis develops. In the next 1-5 days, a creeping eruption evolves as a 2-4 mm wide erythematous, elevated, serpentine track $[2,3]$. Depending on the larval species, larvae migrate several millimeters to a few centimeters per day. Lesions are usually observed on feet, abdomen, buttocks, hands, and genitals, but they can occur on any area of the body [2, 3]. A recent study showed that 81 percent of cutaneous larva migrans patients suffered from severe itching [3].

The diagnosis of cutaneous larva migrans is made on the clinical picture and after a review of the patient's history. A biopsy is not considered useful $[2,3]$. Differential diagnosis include scabies, myasis, tinea corporis, contact dermatitis, or cutaneous pili migrans, but considering the characteristic clinical features of cutaneous larva migrans these conditions can easily be ruled out [3]. The larvae are unable to complete their life cycle in humans and they usually die within several months. Although the character of the disease is self-limiting, the severe itching and risk of secondary infection often warrant therapy [2]. Ivermectin (200 $\mu \mathrm{g} / \mathrm{kg}$, single dose) is the drug of choice in the present case, but other anti-helminthics (e.g., albendazole) are equally effective $[2,3]$. Oral anti-histamines and topical corticosteroids may relieve pruritus. Oral antibiotics are recommended in case of secondary bacterial infection with Staphylococcus or Streptococcus species [2]. Other complications include severe vesiculobullous lesions or pompholyx ( $9 \%$ to $15 \%$ of all cases), such as in the present case. Folliculitis, erythema multiforme, and eosinophilic enteritis may occur. Only very rarely visceral migration of larvae may cause Löffler syndrome $[2,3]$.

Löffler syndrome is characterized by migratory pulmonary infiltrates and peripheral eosinophilia $[1,4,5]$. Respiratory symptoms are minimal or absent and malaise, fever, and cough may be present. In 1946, Wright and Gold reported 26 patients with both cutaneous larva migrans and Löffler syndrome [6]. Del Giudice et al. found only 8 cases published since 1946 [1]. An additional recently reported case shows many similarities with the present case [7]. Infectious causes include infestations with protozoa, fungi, bacteria, or helminthes $[1,4,5]$. Larvae of helminthes have a pulmonary stage in their life cycle and are able to migrate to the lungs. Symptoms of Löffler syndrome are thought to be caused by a type-I allergic reaction during this pulmonary migration of the larvae, resulting in an elevated number of eosinophils in the airways or lung parenchyma, with or without peripheral eosinophilia [1, 4]. Radiographs may show fine miliary nodules or diffuse, reticulonodular interstitial areas of increased opacity. These areas may enlarge as the infestation progresses and sometimes migration of the eosinophil infiltrates are observed on serial radiographs. Eventually, the identification of filariform larvae in the sputum, bronchial washings, or lung biopsy specimens 
may be necessary to diagnose Löffler syndrome [4]. Treatment may include elimination of the larvae with anti-helminthics and oral or inhalation corticosteroids [1]. In a recent case, even intravenous hydrocortisone and oxygen supplementation were necessary to induce remission of Löffler syndrome [7]. Sometimes, however, the signs and symptoms clear spontaneously as well. On the other hand, long-term complications of pulmonary fibrosis and respiratory failure have been reported. A review of the literature regarding Löffler syndrome is depicted in Table 1.

\section{References}

1. Del Giudice P, Desalvador F, Bernard E et al. Loeffler's syndrome and cutaneous larva migrans: a rare association. $\mathrm{Br} \mathrm{J}$ Dermatol. 2002;147(2):386-8. [PubMed]

2. Ghosh SK, Bandyopadhyay D. Dermacase: Can you identify this condition? Cutaneous larva migrans. Can Fam Physician. 2009;55(5):489, 491. [PubMed]

3. Heukelbach J, Feldmeier H. Epidemiological and clinical characteristics of hookworm-related cutaneous larva migrans. Lancet Infect Dis. 2008;8(5):302-9. [PubMed]

4. Andronikou S, du Toit G, Carrighal M, Argent A. Imaging findings in a patient with eosinophilic pneumonia (Loffler's syndrome). SA Journal of Radiology. 2005;9(1):27-9.

5. Chitkara RK, Krishna G. Parasitic pulmonary eosinophilia. Semin Respir Crit Care Med. 2006;27(2):171-84. [PubMed]

6. Wright DO, Gold EM. Loeffler's syndrome associated with creeping eruption (cutaneous helminthiasis). Arch Int Med. 1946;78:303.

7. Tan SK, Liu TT. Cutaneous larva migrans complicated by Löffler syndrome. Arch Dermatol. 2010 Feb;146(2):210-2. [PubMed] 